\title{
THE NUCLEAR STAR CLUSTER OF THE MILKY WAY: STAR FORMATION, STELLAR COLLISIONS AND CENTRAL DARK MASS
}

\author{
R. GENZEL \\ Max-Planck Institut fuer extraterrestrische Physik Garching, \\ $F R G$
}

\begin{abstract}
.
High resolution near-infrared imaging and spectroscopy now gives detailed information about the structure, evolution and mass distribution in the nuclear star cluster of the Milky Way. The central parsec is powered by a cluster of luminous and helium rich, blue supergiants/Wolf-Rayet stars. The most likely scenario for the formation of the massive stars is a star formation burst a few million years ago at which time a dense gas cloud may have fallen into the center. The stellar density in the $\sim 0.3 \mathrm{pc}$ radius central core is high enough that collisions with main sequence stars destroy the largest late type giant stars. Radial velocity measurements for about 300 early and late type stars between 0.1 and $5 \mathrm{pc}$ radius from the dynamic center now strongly favor the existence of a central dark mass of $2.5-3.3 \times 10^{6} M_{\odot}\left(\right.$ density $\left.\left(10^{9} M_{\odot} \mathrm{pc}^{-3}, M / L_{2 \mu \mathrm{m}}\right) \sim 100 M_{\odot} / L_{\odot}\right)$ within $0.1 \mathrm{pc}$ of the dynamic center. This central dark mass cannot be a cluster of neutron stars. It is either a compact cluster of stellar black holes or, most likely, a single massive black hole.
\end{abstract}

\section{INTRODUCING THE PHENOMENA}

The nucleus of the Milky Way (adopted distance $8.5 \mathrm{kpc}$ ) is one hundred times closer to the Earth than the nearest large external galaxy and more than a thousand times closer than the nearest active galactic nuclei. We can therefore study physical processes happening in our own Galactic Center at a level of detail that will never be reached in the more distant, but 
usually also more spectacular systems. What powers these nuclei and how do they evolve? What are the properties of the nuclear stellar clusters? Is star formation happening there? Do dormant massive black holes reside in their cores? In the present pap er I will describe the status of our present knowledge about these key questions. For a more extensive discussion I refer to Genzel,Hollenbach and Townes (1994).

The nuclear mass is dominated by stars, except probably in the innermost parsec. The density of stars increases with decreasing radius $R$ from the dynamic center approximately as $1 / R^{2}$ and attains a value exceeding $10^{6} M_{\odot} / \mathrm{pc}^{3}$ in the central parsec. Infrared observations on a scale of 100 pc to $1 \mathrm{kpc}$ show that these stars appear to be distributed in a rotating bar (Blitz and Spergel 1991). The gravitational torque of this bar may also explain the non-circular motions of interstellar gas clouds found by radio spectroscopy (Binney et al. 1991). The non-circular motions in turn may trigger gas infall into the nucleus. There is increasing evidence from gamma-ray spectroscopy of the $1.8 \mathrm{MeV} 26 \mathrm{Al}$ line (Diehl et al. 1993) and from infrared stellar spectrophotometry (e.g. Cotera et al. 1994, Lebofsky and Rieke 1987) that (massive) star formation has occured throughout the Galactic Center region no longer than 10 million years ago.

Also on a scale of $\sim 100 \mathrm{pc}$ several variable, spectacular hard X-ray and gamma-ray sources have been found (e.g. Skinner 1993). They may represent stellar black holes or neutron stars accreting gas from a companion or from nearby dense gas clouds. Throughout the central few hundred parsecs giant molecular clouds $\left(\sim 10^{6} M_{\odot}\right)$ are found whose gas density $\left(n_{H 2} \sim 10^{4}\right.$ to $10^{6} \mathrm{~cm}^{-3}$ ) and temperature $(40$ to $200 \mathrm{~K})$ are significantly greater than those of the clouds in the Galactic disk (e.g.Guesten 1989). The dynamics of this central molecular cloud layer is characterized by large internal random motions and unusual streaming velocities that can be partially explained by the presence of the central bar potential mentioned above.

Magnetic fields as large as $\sim 1$ mGauss appear to permeate the central $50 \mathrm{pc}$ and are aligned approximately perpendicular to the Galactic plane (e.g.Morris 1993, Sofue 1994). Where they interact with neutral gas clouds remarkable filaments of nonthermal radio synchrotron emission are seen. The central radio rource, SgrA, can be separated into a thermal source, SgrA West and a non- thermal source, SgrA East. SgrA East may be evidence for one or several supernovae that have exploded in the central 10 parsecs within the last $10^{5}$ years (Mezger et al.1989).

While the velocities of gas and stars are approximately constant outside of a few parsec, the velocities are observed to increase within the inner core (e.g. Genzel and Townes 1987). The first evidence for this increase in gas velocities came from mid-infrared spectroscopy of [NeII] by Wollman (1976) and Lacy et al.(1979, 1980). These authors and others following interpreted 
the $>250 \mathrm{~km} / \mathrm{s}$ gas velocities as signalling a concentration of non-stellar mass in the Galactic Center, possibly caused by a few million solar mass black hole at the dynamic center (Lacy et al. 1982, Serabyn and Lacy 1985). However, gas is affected by magnetic, frictional and wind forces, in addition to gravity so that stellar velocities are required to unambiguously determine the mass distribution.

At the dynamic center is a compact radio source, $\mathrm{SgrA}^{*}$, which is close to, but not coincident with a group of bright near-infrared sources (IRS16) of blue color (e.g. Backer 1994). Since its discovery 25 years ago SgrA* has been the most probable candidate for the central black hole. Surrounding the innermost ionized streamers one finds a system of dense orbiting molecular filaments approximately arranged in form of a circum-nuclear 'disk' (Genzel et al. 1985, Jackson et al. 1993). The circum-nuclear disk is probably fed by gas infall from dense molecular clouds at $10 \mathrm{pc}$.

\section{WHAT POWERS THE CENTRAL PARSEC ?}

The observed broad band emission of the central few parsecs is dominated by intense mid- and far-infrared emission from 50 to $100 \mathrm{~K}$ dust grains originating in the circum-nuclear disk and in a cloud ridge associated with the ionized 'mini- spiral'. Taking into account the $(\sim 50 \%)$ fraction of the nuclear radiation not intercepted by the circum-nuclear gas the total UV and visible luminosity of the central parsec has been estimated to lie between 1 and $3 \times 10^{7} L_{\odot}$ (Davidson et al.1992). The Lyman continuum flux is about $2-3 \times 10^{50} \mathrm{~s}^{-1}$ as determined from the thermal radio continuum (Lacy et al. 1980). Infrared spectroscopy of fine structure lines sampling a wide range of excitation stages implies that the effective temperature of the UV radiation field in the central parsec is only about 30,000 to 35,000 $\mathrm{K}$. The line ratios also suggest a heavy element abundance of about twice that in the Sun (Lacy et al. 1980).

What powers this low excitation HII region and what are the properties of the central star cluster? Through the advent of sensitive, large format infrared detector arrays and speckle imaging it has become possible in the last few years to image the central parsec at the resolution to the diffraction limit of $4 \mathrm{~m}$ class telescopes $(\sim 0.1 "$ or $0.04 \mathrm{pc}$ at $2 \mu \mathrm{m}$, Eckart et al. 1992, 1993, 1995). The best current images resolve the near-infrared emission of the central parsec into about 700 stars with K-band $(2.2 \mu \mathrm{m})$ magnitudes $<16$. Thus all red and most blue supergiants, all red giants/AGB stars later than $\mathrm{K} 5$ and all main sequence stars earlier than B0.5 should be detected on those images. The central IRS16 complex located within 1" of the compact radio source SgrA* consists of about two dozen single (or perhaps multiple) stars (see also Simons et al. 1990, Simon et al. 1990). From the number 
distribution of the near-infrared sources it appears that the centroid of the stellar cluster is more likely on SgrA* than on the IRS16 complex and that the core radius of the $\mathrm{K}_{i} 15$ stellar number density distribution is about 0.2 to 0.4 pc (Eckart et al. 1993, 1995, Genzel et al. 1996). If the stars with $\mathrm{K}_{\mathbf{i}} 15$ are representative of the overall mass distribution of the cluster (an assumption that appears very plausible based on recent spectroscopic identification of the stars, Genzel et al. 1996) this core radius together with the mass of stars estimated to lie within a few parsecs indicates that the stellar density in the core about $4-8 \times 10^{6} M_{\odot} \mathrm{pc}^{-3}$. Recent imaging spectroscopy with the new MPE 3D-spectrometer shows that within the core radius bright late type stars (supergiants and the brightest AGB stars) are absent but that the core is surrounded by a ring of red supergiants/AGB stars (Genzel et al. 1996). Following earlier discussions of Lacy et al.(1982), Phinney (1989) and Sellgren et al.(1990), Genzel et al.(1996) interpret this finding in terms of destruction of the brightest (and hence largest) late type giant stars by collisions with main sequence stars. Assuming that such collisions in fact permanently destroy the outer atmosphere of the giants (see Davies et al. 1991) and that collisional destruction becomes observable whenever the collision time is less or equal than the lifetime of the red giant/supergiant phase, the observed lack of stars brighter than $\mathrm{K} \sim 10$ in fact implies a core stellar density of about $5 \mathrm{e} 6 M_{\odot} \mathrm{pc}^{-3}$, in excellent agreement with the density estimated from the number counts.

Another important ingredient of the near-infrared story has been the discovery of a HeI/Brgamma near-infrared emission line star (the AF-star, Forrest et al. 1987, Allen et al. 1990), followed by the discovery of an entire cluster of about 25 such stars in the central parsec and centered on the IRS16/IRS13 complex (Krabbe et al. 1991, 1995). Several of the brightest members of the IRS16 complex are HeI-stars, as is the nearby bright source IRS 13 (Eckart et al. 1995, Krabbe et al. 1995, Libonate et al. 1995, Blum et al. 1995b, Tamblyn et al. 1996). The IRS16 HeI "broad line region" discovered a decade ago by Hall et al. (1982) and Geballe et al. (1984) thus is now identified as a group of luminous mass losing, He-rich stars. Non- local thermodynamic equilibrium (NLTE) stellar atmosphere modeling of the observed emission characteristics of the AF-star (Najarro et al. 1994) confirms and quantifies earlier proposals (Allen et al. 1990, Krabbe et al. 1991) that the AF-star is a WN9/Ofpe star. WN9/Ofpe stars are a rare class of luminous blue supergiants related to luminous blue variables (LBVs), WNL Wolf-Rayet stars and Of/ON supergiants (Allen et al. 1990, Krabbe et al. 1991, Najarro et al. 1994, Libonate et al. 1994, Blum et al. 1995b, Tamblyn et al. 1996). These stars very likely represent the post-main sequence phase of massive stars $\left(20\right.$ to $120 M_{\odot}$ ) before they explode as supernovae. The AF-star has a luminosity of about $3 \times 10^{5} L_{\odot}$, effective temperature near 
$20,000 \mathrm{~K}$ and main-sequence mass between 25 and $40 M_{\odot}$ (Najarro et al. 1994). The surface $\mathrm{He} / \mathrm{H}$ abundance ratio is near unity and the mass loss rate is $6 \times 10^{-5} M_{\odot} \mathrm{yr}^{-1}$ at a velocity of $700 \mathrm{~km} / \mathrm{s}$ (Najarro et al. 1994). Based on the most recent 3D spectroscopy and modelling the brightest $\mathrm{HeI}$ stars (IRS16NE,C,SW, IRS 13) also have effective temperatures between 20,000 and $30,000 \mathrm{~K}$, are helium-rich and are about 5 to 10 times more luminous than the AF star (Krabbe et al. 1995). Their progenitor O stars likely had masses near $100 M_{\odot}$. In addition several stars display CIII/CIV/NIII emission lines, characteristic for late WC and WN Wolf-Rayet stars (Blum et al. 1995, Krabbe et al. 1995, Genzel et al. 1996. Combining the contributions from all its members, the HeI-star cluster can plausibly account for essentially all of the bolometric and Lyman-continuum luminosities of the central parsec (Krabbe et al. 1995). The HeI-star cluster also provides in excess of $10^{38} \mathrm{erg} / \mathrm{s}$ in mechanical wind luminosity which may have a significant impact on the gas dynamics in the central parsec (Genzel, Hollenbach and Townes 1994). Krabbe et al.(1995) successfully fitted the properties of the massive early type stars in the central parsec by a model of a star formation burst between 9 and $3 \times 10^{6}$ years ago in which a few hundred OB stars and perhaps a total of a few thousand stars were formed). This conclusion is in excellent agreement with earlier proposals by Lacy, Townes and Hollenbach (1982), Rieke and Lebofsky (1982) and Allen and Sanders (1986). In the model of Krabbe et al. the HeI stars are the most massive cluster members that in the mean time have evolved off the main sequence and the central parsec is now in the late, wind-dominated phase of the burst. The starburst model accounts naturally for the low excitation of the SgrA (West) HII region. Although there is also evidence for some very young, embedded OB stars the present star formation activity appears to be significantly less than during the peak of the burst. The present gas density in the central parsec is too low for gravitational collapse of gas clouds to stars in the presence of the strong tidal forces (Morris 1993). Perhaps the burst was triggered by infall of a dense gas cloud less than 10 million years ago, a scenario that is supported by an overall counter-rotation (in the sense of Galactic rotation) of the HeI star cluster (Genzel et al. 1996).

Based on earlier theoretical work by Lee (1987), Eckart et al.(1993) have proposed sequential merging by collisions as an alternative to the starburst scenario. A recent Fokker-Planck calculation of an evolving Galactic Center type, dense cluster with merging shows, however, that merging can account for only $\sim 10-20 M_{\odot}$ stars and no $>30 M_{\odot}$ stars (Lee 1994). The basic reason is that in the calculations a sufficiently dense stellar core (density $10^{7} M_{\odot} \mathrm{pc}^{-3}$ or greater) cannot be maintained for a long enough time to build up very many massive stars. Morris (1993) has suggested that the HeI stars are not classical blue supergiants at all but transitory objects 
that have been created in collisions between $\left(\sim 10 M_{\odot}\right)$ stellar black holes and solar mass, red giants. Both accounts of the HeI stars just cited are very specific to the high density environment of the central parsec. However, a number of stars similar to the SgrA HeI stars have now been found in several clusters 2 to 13 ' away from the central, high density SgrA region (Okuda et al. 1990, Moneti et al. 1991, Cotera et al. 1994, Harris et al. 1994, Figer 1995). In the case of the Morris scenario (1993) one probably would also expect a much larger X-ray emission than is observed. These facts and the requirement of having to account for $\sim 100 M_{\odot}$ stars and the presence of heavy element nucleosynthesis products discussed above in my opinion now strongly favors the star formation model over the other scenarios.

There are less than a dozen red supergiants $\left(L>10^{4} L_{\odot}\right)$ in the central starburst zone. Even after correction for collisional desctruction of late type stars mentioned above this suggests that there was relatively little star formation prior to 10-15 million years ago. In comparison there are a much great number of late type stars with luminosities $10^{3}$ to $10^{4} L_{\odot}$, both inside and outside (Haller and Rieke 1989) the central parsec. These medium luminosity stars are likely asymptotic giant branch stars of moderate mass (2 to $7 M_{\odot}$ ). They may signify another starburst episode that happened $\sim 10^{8}$ years ago (Haller and Rieke 1989, Krabbe et al. 1995).

\section{Is SgrA* a Massive Black Hole?}

The next key issue that I want to discuss is the evidence for a central massive black hole. Ever since the original discovery of the nonthermal compact radio source $\mathrm{SgrA}^{*}$ at the core of the nuclear star cluster (Ekers and Lynden-Bell 1971, Downes and Martin 1971, Balick and Brown 1974) that source has been the primary black hole candidate, in analogy to compact nuclear radio sources in other nearby normal galaxies (Lynden-Bell and Rees 1971). In fact ever more detailed radio observations have confirmed the unique nature of SgrA* in the Galaxy. Recent very long baseline interferometry (VLBI) observations at $7 \mathrm{~mm}$ show its size to be less than a few AU (Backer 1994, Krichbaum et al. 1994). Its proper motion relative to a background quasar is now known to be less than about $38 \mathrm{~km} / \mathrm{s}$ (Backer 1994), at least 6 times smaller than the (2d-) velocity dispersion of the stars. Hence $\mathrm{SgrA}^{*}$ must have a mass in excess of about $150 M_{\odot}$. The source shows a $\mathrm{mm} /$ submm excess above the flat $\mathrm{cm}$ - spectral energy distribution (Zylka et al. 1992) probably indicative of the presence of a very compact $\left(\sim 10^{12} \mathrm{~cm}\right)$ radio core of stellar dimensions.

Yet observations at shorter wavelengths indicate nothing particularly impressive toward the radio position SgrA*. The high resolution maps of 
Eckart et al. (1995) for the first time show that SgrA* is located near the centroid of a T-shaped concentration of $\sim 10$ compact near-infrared sources $\left(=3 \mathrm{DSgrA} \mathrm{A}^{*}(\mathrm{IR})\right)$. These sources are likely stars and one might speculate whether they represent a central stellar cusp around SgrA*. SgrA*(IR) also does not show intrinsic variability on scales of minutes or years, or significant line emission (Eckart et al. 1995). Depending on spectral type any possible infrared counterpart of $\mathrm{SgrA}^{*}$ has a luminosity between a few $10^{2}$ and $10^{4} L_{\odot}$. (Variable) hard $\mathrm{X}$ - ray emission is commonly considered a key signature of black holes. However, in contrast to the fairly bright infrared emission the present 1 to $30 \mathrm{keV} \mathrm{X-ray} \mathrm{luminosity} \mathrm{of} \mathrm{SgrA} \mathrm{(West)}$ and SgrA* is less than a a few hundred $L_{\odot}$ (Skinner 1993, Goldwurm et al. 1994). Recent observations with ASCA suggests that SgrA*'s X-ray luminosity may have been larger in the past few hundred years (a few $10^{5} L_{\odot}$, Koyama et al. 1996) but still orders of magnitude smaller than the Eddington rate of a million solar mass black hole (Sunyaev et al. 1993).

The evidence for a (dark) central mass concentration in the Galactic Center thus is based entirely on the gas and stellar dynamics. As mentioned in the Introduction, evidence for a central mass concentration based on gas dynamics had already been growing in the 1980s but had not been considered compelling by most researchers in the field. However, ever better stellar velocities have become available during the past 8 years, fully vindicating the earlier measurements of gas velocities and substantially strengthening the evidence for a compact central dark mass in the Galactic center (Rieke and Rieke 1988, McGinn et al. 1989, Sellgren et al. 1990, Lindqvist et al. 1992, Krabbe et al. 1995, Haller et al. 1996, Genzel et al. 1996).

The most recent determinations by Sellgren et al. (1990), Krabbe et al.(1995), Haller et al.(1996) and Genzel et al.(1996) now are all in good agreement and show a highly significant increase of stellar radial velocity dispersion from about $55 \mathrm{~km} / \mathrm{s}$ at $5 \mathrm{pc}$ to about $180 \mathrm{~km} / \mathrm{s}$ at $0.15 \mathrm{pc}$. From $\sim$ 1"resolution 3D pectroscopy Genzel et al.(1996) have obtained velocities for 222 early and late type stars between 1" and 22" distance from SgrA*. After deprojection of the observed projected velocity dispersions and stellar surface densities Genzel et al.(1996) carried out a Jeans equation analysis. Assuming an isotropic stellar velocity field the new $3 \mathrm{D}$ data in combination with the other stellar measurements mentioned above require a combination of a $\mathrm{M} / \mathrm{L}\left(2\right.$ micron) 2 stellar cluster, and in addition a $2.5-3.3 \times 10^{6} M_{\odot}$ dark mass. The dark compact mass is required at 6 to 8 sigma significance. It can be reduced but not fully removed even if highly anisotropic velocity fields are considered. For comparison, Haller et al.(1996) conclude that there must be a central mass of just under $2 \times 10^{6} M_{\odot}$ and the most recent gas dynamics estimates find a central mass between 2 and $4 \times 10^{6} M_{\odot}$ (Serabyn et al. 1988, Lacy et al. 1991, Herbst et al. 1993). The dark mass is not 
resolved ( $\left.R_{\text {core }}<0.07 \mathrm{pc}\right)$, has a $\mathrm{M} / \mathrm{L}(2$ micron) ratio of at least 100 and a density of $>10^{9} M_{\odot} \mathrm{pc}^{-3}$ (Genzel et al. 1996).

An experiment is now well underway to measure the proper motions of stars between 0.3 " and 10" from SgrA* from repeated high resolution near-infrared imaging with the SHARP camera on the ESO NTT (Eckart and Genzel in prep). This experiment should give a clearcut answer on the anisotropy of the stellar orbits within a few years time.

As the dark mass has a core radius at least 5 times smaller and a core density at least 250 times greater than that of the visible (old) stellar cluster (average stellar mass $\sim 0.7 M_{\odot}$ ) it does not seem plausible that it consists of solar mass remnants (neutron stars or white dwarfs). Calculations of Chernoff and Weinberg (1990) indicate that such large density ratios between similar mass components cannot be attained even in core collapsed globular clusters. The dark mass concentration could either be a single massive black hole, or a very compact cluster of stellar mass $\left(\sim 10 M_{\odot}\right)$ black holes (Morris 1993, Lee 1995) should such a cluster be stable. The most likely configuration is probably a single massive black hole.

If $\mathrm{SgrA}^{*}$ is indeed a million solar mass black hole, the riddle is why it is presently so inactive. It is very interesting that the Galactic Center shares this 'luminosity deficiency' or 'blackness' problem with essentially all nearby nuclei for which there is substantial evidence for dark central masses (Kormendy and Richstone 1995), including the presently most convincing case, the 'mega' $\mathrm{H} 2 \mathrm{O}$ maser source NGC4258 (Myoshi et al. 1995). It is possible that the tidal disruption and accretion of stars by the hole (happening in the Galactic center at a rate of $\sim 10^{-4} \mathrm{yr}^{-1}$ ) occurs very efficiently albeit at low duty cycle (Rees 1988). Accretion of interstellar gas streamers by the hole may be prevented by the need to overcome the angular momentum problem, coupled with the outward force of the stellar winds as discussed above. Finally, the wind gas itself may be accreted largely spherically, with very low radiation efficiency (Melia 1992). A final and very interesting possibility is that most of the energy of the accreting material is advected into the hole and not radiated (Narayan et al. 1995). Nevertheless current models of black hole accretion have to be stretched to be comensurate with $\mathrm{SgrA}^{*}$ being an underfed million solar mass black hole (Ozernoy and Genzel 1996).

\section{Acknowledgements}

I thank the organizers of the conference for a very stimulating meeting and P.Hut and J.Makino for their patience for a very late proceedings contribution. 


\section{References}

Allen, D.A., in Genzel and Harris(1994), 293 (1994)

Allen, D.A., Hyland, A.R. and Hillier, D.J. , MNRAS 244, 706 (1990)

Allen, D.A. and Sanders, R.H., NATURE, 319, 191 (1986)

Backer, D., in Genzel and Harris(1994), 403 (1994)

Balick, B. and Brown, R.L., Ap.J. 194, 265 (1974)

Binney, J.J., Gerhard, O.E., Stark, A.A., Bally, J. and Uchida, K.A.,MNRAS 252, 210 (1991)

Blitz, L. and Spergel, D.N., Ap.J. 379, 631 (1991)

Blum, R.D., Sellgren, K. and dePoy, D.L.. ,Ap.J.440, L17(1995)

Blum, R.D., dePoy, D.L. and Sellgren, K., Ap.J.441, 603 (1995b)

Chernoff, D.F. and Weinberg, M.D., Ap.J. 351, 121 (1990)

Cotera, A.S., Erickson, E.F., Allen, D.A., Colgan, S.W.J., Simpson, J.P.and Burton, M.G., in Genzel and Harris(1994), 217 (1994)

Davidson, J.A., Werner, M.W., Wu, X., Lester, D.F., Harvey, P.M., Joy, M. and Morris, M., Ap.J. 387, 189 (1992)

Davies, M.B., Benz, W. and Hills, J.G., Ap.J. 381, 449 (1991)

Diehl, R. et al., Astr.Ap.(Suppl.) 97, 181 (1993)

Downes, D. and Martin, A., NATURE 233, 112 (1971)

Eckart, A., Genzel, R., Krabbe, A., Hofmann,R. van der Werf, P.P. and Drapatz, S. , NATURE 355, 526(1992)

Eckart, A., Genzel, R., Hofmann, R., Sams, B.J. and Tacconi-Garman, L.E. , Ap.J. 407, L77 (1993)

Eckart, A., Genzel, R., Hofmann, R., Sams, B. and Tacconi-Garman, L.E., Ap.J. 445, L26 (1995)

Ekers, R.D. and Lynden-Bell, D., Ap.Lett. 9, 189 (1971)

Figer, D. , PhD. Thesis, University of California, Los Angeles (1995)

Forrest, W.J., Shure, M.A., Pipher, J.L. and Woodward, C.A.in "The Galactic Center", ed.D.Backer, AIP Conf. Proc 155, 153 (1987)

Geballe, T.R. et al. , Ap.J. 284, 118 (1984)

Genzel, R., Watson, D.M., Crawford, M.K. and Townes, C.H.,Ap.J.297, 766 (1985)

Genzel, R. and Townes, C.H. , Ann.Rev.Astr.Ap. 25, 377(1987)

Genzel, R., Hollenbach, D. and Townes, C.H., Rep.Progr.Phys. 57, 417 (1994)

Genzel, R. and Harris, A.I., Nuclei of Normal Galaxies: Lessons from the Galactic Center, (Dordrecht:Kluwer) (1994)

Genzel, R., Thatte, N., Krabbe, A., Eckart, A., Kroker, H. and Tacconi-Garman, L.E. Ap.J.submitted (1996)

Goldwurm, A. et al., NATURE 371, 5889 (1994)

G-sten, R., in Morris(1989), 89 (1989)

G-sten, R. et al. Ap.J. 318, 124 (1987)

Hall, D.N.B., Kleinmann, S.G. and Scoville, N.Z., Ap.J. 262, L53 (1982)

Haller, J.W. and Rieke, M.J. in Morris(1989), 487 (1989)

Haller, J.W., Rieke, M.J. and Rieke, G.H., Tamblyn, P., Close, L. and Melia, F., Ap.J. 456, 194 (1996)

Harris, A.I. et al., in Genzel and Harris (1994), 223 (1994)

Herbst, T.M., Beckwith, S.V.W., Forrest, W.J. and Pipher, J.L., A.J. 105, 956 (1993)

Jackson, J. et al., Ap.J. 402, 173 (1993)

Kormendy, J. and Richstone, D., Ann.Rev.Astr.Ap.33, 581 (1995)

Koyama, K.,et al. preprint (1995)

Krabbe, A., Genzel, R., Drapatz, S. and Rotaciuc, V., Ap.J. 382, L19 (1991)

Krabbe, A. Genzel, R., Eckart, A., Najarro, F., Lutz, D. et al., Ap.J.Lett. 447, L95 (1995)

Krichbaum, T.P., Schalinski, C.J., Witzel, A., Standke, K.J., Graham, D.A and Zensus, J.A.in Genzel and Harris(1994), 411 (1994)

Lacy, J.H., Baas, F., Townes, C.H. and Geballe, T.R. Ap.J. 227, L17 (1979) 
Lacy, J.H., Townes, C.H., Geballe, T.R. and Hollenbach, D.J., Ap.J. 241, 132 (1980)

Lacy, J.H., Townes, C.H. and Hollenbach, D.J., Ap.J. 262, 120 (1982)

Lacy, J.H., Achtermann, J.M. and Serabyn, E., Ap.J. 380, L71 (1991)

Lebofsky, M.J. and Rieke, G.H., in The Galactic Center, ed. D.Backer, American Institute of Physics Conf.Proc.155, 79 (1987)

Lee, H.M. Ap.J. 319, 801 (1987)

Lee, H.M., in Genzel and Harris(1994), 335 (1994)

Lee, H.M., MNRAS, 272, 605 (1995)

Libonate, S., Pipher, J.L., Forrest, W.J. and Ashby, M.L.N., Ap.J. 439, 202 (1995)

Lindqvist, M., Habing, H. and Winnberg, A., Astr.Ap. 259, 118 (1992)

Lo, K.Y. and Claussen, M, J. , NATURE 306, 647 (1983)

Lynden-Bell, D. and Rees, M., MNRAS 152, 461 (1971)

Melia, F. ,Ap.J. 387, L25 (1992)

Mezger, P.G.et al. 1989, Astr.Ap. 209, 337

Mezger, P.G., in Genzel and Harris(1994), 415 (1994)

Miyoshi,M, Moran, J.M., Hernstein, J., Greenhill, L., Nakai, N., Diamond, P. and Inoue, M., NATURE 373, 127 (1995)

Moneti, A., Glass, I.,S. and Moorwood, A.F.M. , Mem.Soc.Astr.Ital.62, 4,755 (1991)

Morris, M. ,in Galactic and Extragalactic Magnetic Fields, eds. R.Beck, P.Kronberg and R.Wielebinski (Dordrecht:Kluwer), 361 (1990)

Morris, M., Ap.J . 408, 496 (1993)

Morris, M. (ed.), "The Center of the Galaxy" (Dordrecht:Kluwer) (1989)

Najarro, F. et al., Astr.Ap. 285, 573 (1994)

Narayan, R., Yi, I., and Mahadevan, R., NATURE 374, 623 (1995)

Okuda, H. et al. , Ap.J.351, 89 (1990)

Ozernoy, L. and Genzel, R., in "The Galaxy", ed.L.Blitz (Dordrecht:Kluwer), in press (1995)

Phinney, E.S., in Morris(1989), 543 (1989)

Rees, M., NATURE 333, 523 (1988)

Rieke, G. and Rieke, M. in Genzel and Harris(1994), 283 (1994)

Rieke, G.H. and Lebofsky, M.J., in "The Galactic Center", eds. G.Riegler and R.D.Blandford, AIP conf. proc. 83 (New York), 194 (1982)

Rieke, G.H. and Rieke, M.J., Ap.J. 330, L33 (1988)

Sellgren, K., McGinn, M.T., Becklin, E. and Hall, D.N.B., Ap.J. 359, 112 (1990)

Serabyn, E. and G-sten, R. , Astr.Ap. 184, 133 (1987)

Serabyn, E. and Lacy, J., Ap.J. 293, 445 (1985)

Serabyn, E., Lacy, J., Townes, C.H. and Bharat,R., Ap.J. 326, 171 (1988)

Skinner, G.K., Astr.Ap.(Suppl.) 97, 149 (1993)

Simon, M. et al. Ap.J. 360, 95 (1990)

Simons, D.A., Hodapp, K.W. and Becklin, E.E. Ap.J. 360, 106 (1990)

Sofue, Y., in Genzel and Harris (1994), 43 (1994)

Sunyaev, R.A., Markevitch, M. and Pavlinsky, M., Ap.J. 407, 606 (1993)

Tamblyn, P., Rieke, G.H., Hanson, M.M., Close, L.M., McCarthy, D.W. and Rieke, M.J., Ap.J. 456, 206 (1996)

Wollman, E., Ph.D. Thesis ,Univ.of California, Berkeley, (1976)

Zylka, R., Mezger, P.G. and Lesch, J. , Astr.Ap.261, 119 (1992) 